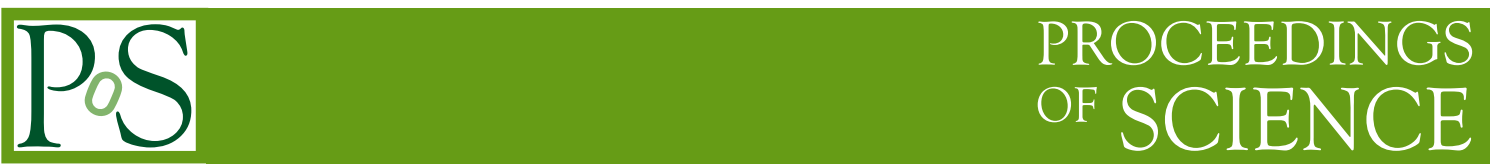

\title{
Refurbishment of KamLAND Outer Detector
}

\section{H. Ozaki* and J. Shirai}

On behalf of the KamLAND Collaboration

Research Center for Neutrino Science, Tohoku University, Japan

E-mail: ozaki@awa.tohoku.ac.jp

KamLAND, a large size liquid scintillator detector for anti-neutrino detection, has observed the reactor neutrino oscillation and geo-neutrinos since its operation started in 2002. The outer detector (OD) is a water Cherenkov detector with 3,200 $\mathrm{m}^{3}$ of water and 225 Kamiokande-PMTs serving as the veto counter against the cosmic-ray muons. Muon tagging efficiency of the OD has been decreasing since 2010 because of a gradual increase of PMT failures, which results in the increase of muon-induced backgrounds. We have carried out a refurbishment of the OD from January to April in 2016 by replacing the PMTs and improving light collection in the less-sensitive equator region. As a result, we achieved a recovery of the OD detection efficiency of muons equal to $99.8 \%$.

38th International Conference on High Energy Physics 3-10 August 2016

Chicago, USA

\footnotetext{
* Speaker.
} 


\section{Introduction}

KamLAND (Kamioka Liquid-scintillator AntiNeutrino Detector) is located 1,000 m deep underground in the Kamioka-mine in Japan. As shown in Figure 1, there are two main parts in KamLAND. The inner detector (ID) is a liquidscintillator detector equipped with 1325 17-inch PMTs and 554 20-inch PMTs on the inner wall of the spherical stainless steel tank of $18 \mathrm{~m}$ in diameter. The spherical ballon of $13 \mathrm{~m}$ in diameter, constructed of nylon and EVOH films, is hanged in the ID tank and holds $1,200 \mathrm{~m}^{3}$ of ultra-pure liquid scintillator. The outer space of the balloon is filled with non-scintillating buffer oil to reduce backgrounds from the PMTs and other external

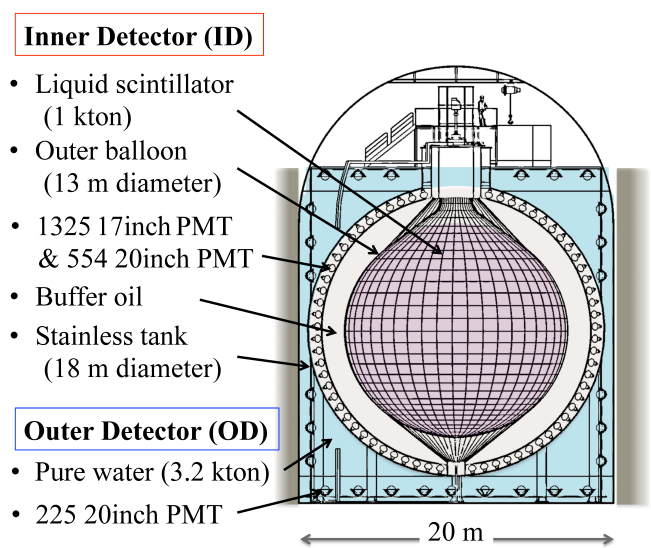

Figure 1: KamLAND Detector Schematic materials. The outer detector (OD) is a water Cherenkov detector, which consists of 3,200 $\mathrm{m}^{3}$ of purified water and 225 Kamiokande 20-inch PMTs. The surface of the OD cylindrical cavern and the ID spherical tank are covered with reflective Tyvek1073B sheets in order to enhance the light collection efficiency to the PMTs. The purposes of the OD are the veto of muons, the shielding of the radioactivity from external rocks, and the control of the ID temperature.

KamLAND experiment has many physics targets, such as anti-neutrinos from reactors, the Earth, and the universe, and neutrinos from supernovae and the Sun. We achieved the first observation of the reactor neutrino oscillation in 2002 [1] and the first detection of geo-neutrinos in 2005 [2]. In 2011, we installed a mini-balloon of 3.1m diameter in the center of the outer balloon, containing Xe (enriched with ${ }^{136} \mathrm{Xe}$ ) loaded liquid scintillator and started a neutrino-less double beta decay search for ${ }^{136} \mathrm{Xe}$ nuclei (KamLAND-Zen). We obtained the most stringent upper limit on the effective Majorana neutrino mass in 2016 [3].

Since the start of KamLAND, the number of the bad OD PMTs whose hit rates are extremely low has been gradually increasing and reached more than $60 \%$. As a result, the OD detection efficiency, estimated by muons going through the ID, had been decreasing since 2010. If the OD misses muons, muon-induced products entering the ID would cause background events. For example, by the prompt proton recoil and the delayed thermal neutron capture events, muon-induced fast neutron can satisfy the delayed coincidence criteria for electron anti-neutrino selection. In addition, the narrow space between the rocks and the equator region of the spherical tank has limited the PMT arrangement, resulting in the lower detection efficiency. We found that in the equator region there are more muon-induced background events not detected by the OD and that intrude into the ID. Our goal of the OD refurbishment is to restore the OD detection efficiency by mainly replacing all the PMTs, and improving the performance of the equator region of the OD.

\section{Refurbishment of OD}

We began the work on the OD refurbishment in Jan. 5th, 2016 and restarted the OD DAQ 
after filling the OD with water in Apr. 21st, 2016. During the work, we took the ID data only with 17-inch PMTs after working hours in order not to miss the chance of a supernova detection. The numbers and arrays of the PMTs for the new OD were determined by simulation studies on cosmicray muon and radon decay events. The contents of the refurbishment are summarized below.

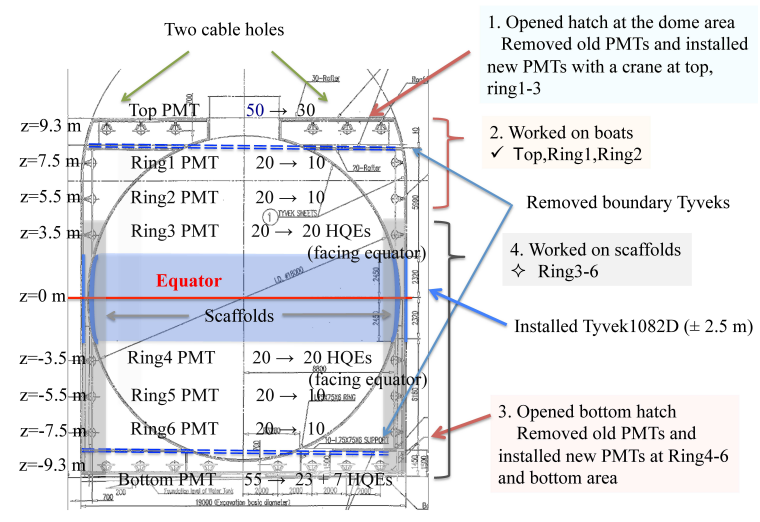

Figure 2: Refurbishment works
Table 1: PMT replacement summary

\begin{tabular}{lc|cc|cc}
\hline & & \multicolumn{2}{|c|}{ Before } & \multicolumn{2}{c}{ After } \\
\hline & $z[\mathrm{~m}]$ & $\begin{array}{c}\text { \# of } \\
\text { PMTs }\end{array}$ & $\begin{array}{c}\text { Trigger } \\
\text { section }\end{array}$ & $\begin{array}{c}\text { \# of } \\
\text { PMTs }\end{array}$ & $\begin{array}{c}\text { Trigger } \\
\text { section }\end{array}$ \\
\hline Top & 9.3 & 50 & top & 30 & top \\
Ring1 & 7.5 & 20 & upper & 10 & top \\
Ring2 & 5.5 & 20 & upper & 10 & middle \\
Ring3 & 3.5 & 20 & upper & $20 \mathrm{HQE}$ & middle \\
Ring4 & -3.5 & 20 & lower & $20 \mathrm{HQE}$ & middle \\
Ring5 & -5.5 & 20 & lower & 10 & middle \\
Ring6 & -7.5 & 20 & lower & 10 & bottom \\
Bottom & -9.3 & 55 & bottom & $23+7 \mathrm{HQE}$ & bottom \\
\hline
\end{tabular}

- Photomultiplier tube replacement: We removed all 225 Kamiokande PMTs and installed 140 new 20-inch PMTs (R3600-06MOD) produced by Hamamatsu. See Figure 2. 47 HQEtypes (Quantum Efficiency is about 30\% at $400 \mathrm{~nm}$ wavelength) were set on Ring3, Ring4 and Bottom. The number of PMTs on the Ring 3 and Ring 4 were kept at 20 and they are facing the equator region by tilting them $11^{\circ}$ outward from the vertical direction. The arrangements of the PMTs are summarized in Table 1. Each PMT is equipped with a hemispherical mesh and a cone of $\mu$-metal which was reused for the shielding against the Earth's magnetic field. The photo-coverage of the PMTs after refurbishment is $0.92 \%$.

- New highly reflective Tyvek: We found that Tyvek1073B becomes transparent in spots by the long term use in water. We placed high-reflectivity sheets, Tyvek1082D $(260 \mu \mathrm{m}$ thickness) [4] on the old Tyvek1073B in the equator region from $z=-2.5 \mathrm{~m}$ to $z=2.5 \mathrm{~m}$ to improve the reflection efficiency of the Cherenkov light as much as possible. The sheets were chosen by a bench-top experiment with several types of candidate sheets tested.

- Boundary Tyvek removal: We removed the boundary Tyvek sheets at $z=-8.5 \mathrm{~m}$ and $z=8.5 \mathrm{~m}$ to further improve the detection efficiency of the Cherenkov light.

- Trigger hardware update: The OD trigger system has also been upgraded as seen in Table 1. Originally, there were 4 sections separated by the Tyvek sheets: top, upper, lower and bottom. The trigger thresholds were set by the number of PMT hits in each section. After the refurbishment, three new sections: top, middle, and bottom were installed according to the optimization suggested by the OD simulation. Moreover, a new electronics module was introduced to sum up the PMT hits of all 3 sections for a new trigger signal defined by the hit sum of all 140 PMTs. The new trigger system was installed in August 2016.

- Others: Other works during the refurbishment included installation of new thermometers and a water-level meter, inspection of the spherical tank, and repair work for water leakage. 


\section{Analysis}

The OD detection efficiency of cosmic-ray muons after the refurbishment is estimated by muons going through the ID. The incident angles and the kinetic angles of muon tracks are obtained by muon event reconstruction in the ID, and muons missed by the OD are plotted in Figure 3. The figure shows that the OD detection efficiency for muons penetrating the equator region is significantly improved after the refurbishment. There are more muons not detected by the OD after the refurbishment around the chimney region (top region of the detector) due to the 8-inch PMTs in the region (not shown in the figures) not being used yet. However, these muons are detected by the ID and easily identified by the large number of emitted photons. The fraction of undetected muons going through the ID decreased from $\sim 0.3 \%$ just before the refurbishment to $\sim 0.2 \%$ after the upgrade was completed. In the equator region, the improvement is more significant. This fraction is decreased from $1.10 \pm 0.02 \%$ to $0.24 \pm 0.01 \%$.
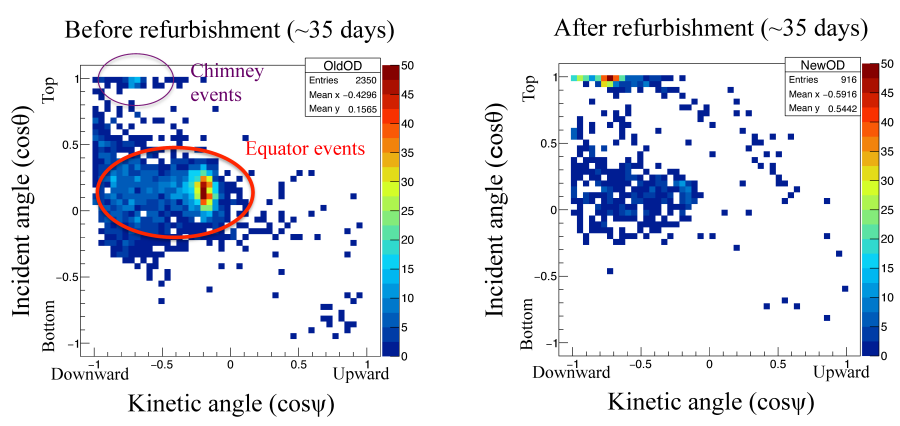

Kinetic angle $(\cos \psi)$
Figure 3: Muon events missed by the OD. Left panel is the data just before and right panel is after the refurbishment. The kinetic angle is the zenith angle of the muon track, and the incident angle is the zenith angle of the muon entrance point to the ID. The central cluster corresponds to equator muons and the events at the top region in the figure correspond to chimney muons.

\section{Summary}

We refurbished the KamLAND outer Cherenkov detector. All 225 old PMTs were removed and 140 new PMTs were installed. The HQE PMTs and the new reflection sheets were installed to reinforce the detection efficiency in the equator region. The improvement of the OD detector performance is verified by muons going through the ID and a much better performance in the equator region is found. This result suggests that muon-induced backgrounds are expected to be significantly suppressed by the OD in the future measurements.

\section{References}

[1] K.Eguchi et al, "First Results from KamLAND: Evidence for Reactor Antineutrino Disappearance", 10.1103/PhysRevLett.90.021802, pp.1-6, January 2003.

[2] T.Araki et al, "Experimental investigation of geologically produced antineutrinos with KamLAND", Nature 436,449 (2005).

[3] A.Gando et al, "Search for Majorana Neutrinos Near the Inverted Mass Hierarchy Region with KamLAND-Zen”, 10.1103/PhysRevLett.117.082503, pp.1-6, August 2016.

[4] L.Wang et al, "Study of Tyvek reflectivity in water", Chin.Phys.C, vol36(2012), pp.628-632. 